After transplantation the immunosuppressive agents azathioprine and prednisone or prednisolone were used. Treatment of rejection involved a massive increase in administration of steroids, and in some cases actinomycin $C$, extracorporeal irradiation or antilymphoeytic globulin were used.

The most satisfactory cases were discharged from hospital within 6 wecks of surgery, but the average post-operative period in hospital was 9 weoks. An attempt was made to see all patients every 3 months at Cambridge.

The results of the follow-up show that of the three patients who received kidneys from their parents, two are alive and well after 3 months and 2 weeks respectively, and have good transplant function; one died after 4 weeks. Results for patients receiving kidneys from cadavers are shown in the table. Five of these patients each received two kidneys.

Transplants in 42 patients after 4 or more months Functioning transplants 25 of 44 (58 per cent)

At 4 months 17 of 29 ( 59 per cent) Living patients

At 1 moar 17 of 29 ( 59 per cent)
6 of 7 ( 86 per cent) 28 of 42 ( 67 per cent) 17 of 27 ( 633 per cent) 6 of 7 ( 86 per cent)

Of the nineteen transplants that failed, rejection occurred in nine cases, but a number of other complications also developed in some patients. Five patients received second transplants, and the cases transplanted during the past 4 months are too early to assess. Twenty-four patients have been restored to normal activity, and most of them have returned to their previous jobs. If it is remembered that before surgery these patients existed in a moribund condition, these results from Addenbrooke's Hospital suggest that the risk of renal transplantation is on the whole worth taking.

\section{New Polymers}

A symposium was held at the University of Manchester on April 8 and 9 to review advances in the field of new polymeric materials. The main contributions were presented by eight invited speakers.

Professor C. S. Marvel (University of Arizona) discussed attempts to find thermally stable polymers capable of maintaining their physical properties at temperatures of at least $300^{\circ} \mathrm{C}$ in air. In general, the organic polymers which will withstand these temperatures are aromatic structures, usually heterocyclic, with a low hydrogen content. The 'Viton' rubbers (copolymers of vinylidene fluoride and hexafluoropropylene) are the first of these materials to go into smallscale commercial production. Most polymers are insulators, but structures are possible which will produce the electrically conductive polymers discussed by Professor H. A. Pohl (University of Oklahoma). A system of conjugated double bonds extending throughout a rigid macromolecule has a high conductivity limited only by the gaps separating the ends of molecules. The effect of this energy barrier can be reduced by applying high pressures to the system.

Professor R. N. Haszeldine (Institute of Science and Technology, University of Manchester) gave a review of the preparation of nitroso-elastomers which have excellent low temperature properties and outstanding inertness both to chemicals and solvents.
Professor O. Wichterle (Macromolecular Institute. Prague), described recent attempts to prepare polyconjugated polymers. In the past the basic reason for the failure to obtain continuous conjugation along the backbone chain of a macromolecule has been the lack of stabilization of the transition structures occurring in the polyconjugation growth. In Prague the stabilization of living acetylene polymers has been achieved. The anionic polymerization, in polar solvents (for example, dimethylsulphoxide), of acetylene, dicyanoacetylene and cyanogen is also possible.

Of the quarter million or so polymers and copolymers which came within the scope of his review, Dr 1. Goodman (ICL P and P Laboratory) discussed those polyesters and polyamides which are of commercial interest. Lactones are becoming increasingly important as sources of polyesters: also polyesters containing aliphatic or aromatic rings in the chain backbone other than poly(ethylene terephthalate) are reaching the market. for example, poly $(c$-hexane $1: 4$ dimethylene (terephthalate). Nylons 6 and $6 \cdot 6$ still dominate the polyamide field and are unlikely to be replaced on technical grounds: the improved thermal stability and lower moisture absorption of the higher nylons (7--12) are counteracted by their lower moduli, especially at high temperatures.

Professor Morton (University of Akron) reported the controlled synthesis of polymers containing blocks of polystyrene and polydiene (butadiene or isoprene) of structure SDS. In these materials, a dispersion of incompatible polystyrene domains which are glassy below $70^{\circ} \mathrm{C}$ act as cross-links. The stress-strain properties of these thermoplastic elastomers are not affected by the chain lengths of monodisperse diene blocks in the range $600-2,000$ diene units, but the polydispersity of the block lengths does influence these properties.

Two aspects of composite materials were presented at the last session, first by Dr J. Economy (Carborundum Co., Niagara Falls, USA), who spoke about the preparation and properties of novel boron nitride and graphite fibres, and second by Dr A. Kelly (National Physical Laboratory), who discussed the principles involved in reinforcing polymers with these novel fibrous materials.

Overall, the impression gained was that there were no new materials available which are likely to competc in quantity with the well established polymers. On the other hand, special-purpose polymers, such as the benzimidazoles, are in small scale production despite their cost and the serious problems of fabrications. The block copolymer rubbers are scientifically interest. ing but still have to make a technological impact. Perhaps the most exciting possibilities lie in the field of composites, where there is much pioneering work to be done both in methods of preparation and in the establishment of the principles of reinforcement.

\section{Two-fluid Solar Wind}

A THEORY of the solar wind which purports to take full account of the separate existence of protons and electrons in the outwards stream of particles from the Sun has now been published by R. E. Hartle and P. A. Sturrock of the Ames Research Center of NASA (Astrophysical J., 151, 1155; 1968). The starting point for the argument is that the rate of exchange of 